\title{
Application of Market Models to Network Equilibrium Problems
}

\author{
I. V. Konnov1
}

\begin{abstract}
We present a general two-side market model with divisible commodities and price functions of participants. A general existence result on unbounded sets is obtained from its variational inequality re-formulation. We describe an extension of the network flow equilibrium problem with elastic demands and a new equilibrium type model for resource allocation problems in wireless communication networks, which appear to be particular cases of the general market model. This enables us to obtain new existence results for these models as some adjustments of that for the market model. Under certain additional conditions the general market model can be reduced to a decomposable optimization problem where the goal function is the sum of two functions and one of them is convex separable, whereas the feasible set is the corresponding Cartesian product. We discuss some versions of the partial linearization method, which can be applied to these network equilibrium problems.

Key words: Market models; divisible commodities; price functions; variational inequality; existence results; partial linearization; component-wise steps; network flow equilibria; elastic demands; wireless communication networks.
\end{abstract}

\section{Introduction}

Investigation of complex systems with active elements having their own interests and sets of actions is usually based on a suitable equilibrium concept. Such a concept should equilibrate different interests and opportunities of the elements (agents, participants) and provide ways of its proper implementation within some accepted basic (information) behavior framework of the system under investigation.

For instance, the classical perfectly (Walrasian) and imperfectly (Cournot - Bertrand) competitive models, which are most popular in economics (see e.g. [1, 2] and references therein), reflect different equilibration mechanisms and information frameworks. We recall that actions of any separate agent within a perfect competition model can not impact the state of the whole system, hence any agent may utilize some integral system parameters (say, prices), rather than the information about the behavior of other agents. On the contrary, actions of any separate agent in an imperfectly competitive model can change the state of the whole system. Therefore, the model

\footnotetext{
${ }^{1}$ Department of System Analysis and Information Technologies, Kazan Federal University, ul. Kremlevskaya, 18, Kazan 420008, Russia. E-mail: konn-igor@ya.ru
} 
is formulated as a (non-cooperative) game problem and is usually based on the wellknown Nash equilibrium concept [3]. Nevertheless, real systems (markets) may give wide variety of these features and different information frameworks. Hence, flexible equilibrium models could also be very useful for derivation of efficient decisions in complex systems.

In this paper, we consider a general two-side market model with divisible commodities and price functions of participants. It is based on the auction market models proposed in [4, 5], where the equivalence result with a variational inequality problem was established. Afterwards, some extensions to the multi-commodity case and applications to resource allocation in telecommunication networks were suggested in [6, 7]. The alternative equilibrium concept related to this model was proposed in [8], where it was also shown that the same equilibrium state can be attained within different mechanisms and information exchange schemes, including the completely decentralized competitive mechanism.

We now suggest a somewhat more general class of market equilibrium models, which follows the approach from [8]. It is subordinated to the material balance condition and can also be formulated as a variational inequality problem, hence one can utilize the well-developed theory and methods of variational inequalities for investigation and solution finding of this equilibrium model. We give a new existence result for the model in the case where the feasible set is unbounded. Besides, under certain integrability conditions the model can be also reduced to an optimization problem. We suggest a new cyclic version of the partial linearization method for its decomposable case. We describe extensions of the known network flow equilibrium problem with elastic demands and a resource allocation problem in wireless communication networks and show they are particular cases of the presented market model. This enables us to obtain new existence results for these models and to solve these problems with the partial linearization method.

\section{A general multi-commodity market equilibrium model}

We start our considerations from a general market model with $n$ divisible commodities, which somewhat extends those in [6, 8]; see also [9] for the vector model. For each commodity $s$, each trader $i$ chooses some offer value $x_{i s}$ in his/her capacity segment $\left[\alpha_{i s}^{\prime}, \alpha_{i s}^{\prime \prime}\right]$ and has a price function $g_{i s}$. Similarly, each buyer $j$ chooses some bid value $y_{j s}$ in his/her capacity segment $\left[\beta_{j s}^{\prime}, \beta_{j s}^{\prime \prime}\right]$ and has a price function $h_{j s}$. We denote by $I_{s}$ and $J_{s}$ the finite index sets of traders and buyers attributed to commodity $s$ and set $N=\{1, \ldots, n\}$. Clearly, each trader/buyer can be attributed to many commodities. We suppose that the prices may in principle depend on all the bid/offer volumes of all the commodities. That is, if we set $x_{(s)}=\left(x_{i s}\right)_{i \in I_{s}}, x=\left(x_{(s)}\right)_{s \in N}, y_{(s)}=\left(y_{j s}\right)_{j \in J_{s}}$, $y=\left(y_{(s)}\right)_{s \in N}$, and $w=(x, y)$, then $g_{i s}=g_{i s}(w)$ and $h_{j s}=h_{j s}(w)$. Let $b_{s}$ denote the 
value of the external excess demand for commodity $s$, then $b=\left(b_{s}\right)_{s \in N}$. If it equals zero, the market is closed. Any market solution must satisfy the balance equation, hence we obtain the feasible set of offer/bid values

$$
\begin{aligned}
& W=\prod_{s \in N} W_{s}, \text { where } \\
& W_{s}=\left\{\begin{array}{l|l}
w_{(s)}=\left(x_{(s)}, y_{(s)}\right) & \begin{array}{l}
\sum_{i \in I_{s}} x_{i s}-\sum_{j \in J_{s}} y_{j s}=b_{s} ; \\
x_{i s} \in\left[\alpha_{i s}^{\prime}, \alpha_{i s}^{\prime \prime}\right], i \in I_{s}, y_{j s} \in\left[\beta_{j s}^{\prime}, \beta_{j s}^{\prime \prime}\right], j \in J_{s}
\end{array}
\end{array}\right\} ; \\
& \text { for } s \in N \text {. }
\end{aligned}
$$

A vector $\bar{w}=(\bar{x}, \bar{y}) \in W$ is said to be a market equilibrium point if there exists a price vector $\bar{p}=\left(\bar{p}_{s}\right)_{s \in N}$ such that

$$
g_{i s}(\bar{w})\left\{\begin{array}{lll}
\geq \bar{p}_{s} & \text { if } \quad \bar{x}_{i s}=\alpha_{i s}^{\prime}, \\
=\bar{p}_{s} & \text { if } \quad \bar{x}_{i s} \in\left(\alpha_{i s}^{\prime}, \alpha_{i s}^{\prime \prime}\right), \quad \text { for } i \in I_{s} ; \\
\leq \bar{p}_{s} & \text { if } \quad \bar{x}_{i s}=\alpha_{i s}^{\prime \prime},
\end{array}\right.
$$

and

$$
h_{j s}(\bar{w})\left\{\begin{array}{lll}
\leq \bar{p}_{s} & \text { if } \quad \bar{y}_{j s}=\beta_{j s}^{\prime}, \\
=\bar{p}_{s} & \text { if } \quad \bar{y}_{j s} \in\left(\beta_{j s}^{\prime}, \beta_{j s}^{\prime \prime}\right), \quad \text { for } j \in J_{s} ; \\
\geq \bar{p}_{s} & \text { if } \quad \bar{y}_{j s}=\beta_{j s}^{\prime \prime},
\end{array}\right.
$$

for $s \in N$. We now give the basic relation between the market equilibrium problem (11) -(2) and a variational inequality (VI, for short). Its proof is almost the same as that in [6, Theorem 2.1] and is omitted.

Proposition 2.1 (a) If $(\bar{w}, \bar{p})$ satisfies (1)-(2) for $s \in N$ and $\bar{w} \in W$, then $\bar{w}$ solves $V I:$ Find $\bar{w} \in W$ such that

$$
\sum_{s \in N}\left[\sum_{i \in I_{s}} g_{i s}(\bar{w})\left(x_{i s}-\bar{x}_{i s}\right)-\sum_{j \in J_{s}} h_{j s}(\bar{w})\left(y_{j s}-\bar{y}_{j s}\right)\right] \geq 0 \quad \forall w \in W .
$$

(b) If a vector $\bar{w}$ solves VI (3), then there exists $\bar{p} \in \mathbb{R}^{n}$ such that $(\bar{w}, \bar{p})$ satisfies (11)-(2) for $s \in N$.

The presence of the price functions is invoked by complexity of the whole system, i.e., the price functions may contain participants' intentions or reflect interdependence (mutual influence) of the elements, which need not be known to the participants.

It follows from Proposition 2.1 that we can establish existence results for equilibrium problems of form (11)-(2) by using suitable results from the theory of VIs or more general equilibrium problems. For instance, if the feasible set $W$ is bounded and the cost mapping of VI (3) is continuous, then equilibrium problem (11)-(2) has a solution. In the unbounded case, we need certain coercivity assumptions. 
We follow the approach from [, 9] and consider for simplicity the case where all the lower bounds $\alpha_{i s}^{\prime}$ and $\beta_{j s}^{\prime}$ of capacities are fixed and greater than $-\infty$, whereas some upper bounds $\alpha_{i s}^{\prime \prime}$ and $\beta_{j s}^{\prime \prime}$ can be absent. Then, for each commodity $s \in N$, we define the index sets

$$
I_{s}^{u}=\left\{i \in I_{s} \mid \alpha_{i s}^{\prime \prime}=+\infty\right\} \text { and } J_{s}^{u}=\left\{j \in J_{s} \mid \beta_{j s}^{\prime \prime}=+\infty\right\},
$$

and take the following coercivity condition.

(C) There exists a number $r>0$ such that for any point $w=(x, y) \in W$ and for each $s \in N$ it holds that

$$
\begin{aligned}
& \forall l \in J_{s}^{u}, y_{l s}>\max \left\{r, \beta_{j s}^{\prime}\right\} \Longrightarrow \exists k \in I_{s}^{u} \text { such that } \\
& x_{k s}>\alpha_{k s}^{\prime} \text { and } g_{k s}(w) \geq h_{l s}(w) .
\end{aligned}
$$

This condition seems rather natural: at any feasible point $w$ and for each fixed commodity $s$, any large demand value of buyer $l$ invokes existence of a trader $k$ whose price is not less than the price of buyer $l$.

Proposition 2.2 Suppose that the set $W$ is nonempty, all the functions $g_{i s}$ and $g_{j s}$ are continuous for all $i \in I, j \in J$, and $s \in N$. If condition (C) is fulfilled, then VI (3) has a solution.

The proof of this assertion is almost the same as those in [8, Theorem 1] and [9, Theorem 4.3] and is omitted.

\section{Partial linearization methods}

Due to Proposition 2.1, we can take various iterative solution methods for optimization and variational inequality problems (see e.g. [5, 8, 10]) for finding solutions of the market equilibrium problems of form (11)-(2). We now intend to consider a special integrable class of these problems that admits efficient iterative solution methods.

Let us first take a problem of minimization of the sum of two functions $\mu(w)+\eta(w)$ over a feasible set $W \subseteq \mathbb{R}^{m}$, or briefly,

$$
\min _{w \in W} \rightarrow\{\mu(w)+\eta(w)\} .
$$

We suppose that the set $W \subset \mathbb{R}^{m}$ is non-empty, convex, and compact, both the functions are convex and $\mu: \mathbb{R}^{m} \rightarrow \mathbb{R}$ is smooth. Moreover, the minimization of the function $\eta$ over the set $W$ is not supposed to be difficult. In this case one can apply the partial linearization (PL for short) method, which was first proposed in [11].

\section{Method (PL).}

Choose a point $w^{0} \in W$ and set $k=0$. At the $k$-th iteration, $k=0,1, \ldots$, we have a point $w^{k} \in W$. Find some solution $v^{k}$ of the problem

$$
\min _{v \in W} \rightarrow\left\{\left\langle\mu^{\prime}\left(w^{k}\right), v\right\rangle+\eta(v)\right\}
$$


and define $p^{k}=v^{k}-w^{k}$ as a descent direction at $w^{k}$. Take a suitable stepsize $\lambda_{k} \in(0,1]$, set $w^{k+1}=w^{k}+\lambda_{k} p^{k}$ and $k=k+1$.

The stepsize can be found either with some one-dimensional minimization procedure as in [11] or with an inexact Armijo type linesearch; see also [12, 13] for substantiation and further development.

The usefulness of this approach becomes clear if problem (44) is (partially) decomposable, which is typical for very large dimensional problems. For instance, let

$$
\eta(w)=\sum_{s \in N} \eta_{s}\left(w_{(s)}\right) \text { and } W=\prod_{s \in N} W_{s},
$$

where $w_{(s)} \in W_{s} \subset \mathbb{R}^{m_{s}}$, so that $m=\sum_{s \in N} m_{s}$, i.e., there is some concordant partition of the initial space $\mathbb{R}^{m}$. Then we have the problem

$$
\min _{w \in \prod_{s \in N} W_{s}} \rightarrow\left\{\mu(w)+\sum_{s \in N} \eta_{s}\left(w_{(s)}\right)\right\}
$$

and (5) becomes equivalent to several independent problems of the form

$$
\min _{v_{(s)} \in W_{s}} \rightarrow\left\{\left\langle v_{(s)}, \frac{\partial \mu\left(w^{k}\right)}{\partial w_{(s)}}\right\rangle+\eta_{s}\left(v_{(s)}\right)\right\} ;
$$

for $s \in N$. The above descent method admits various component-wise iterative schemes; see e.g. [14.

Our market equilibrium problem from the previous section written as VI (3) is reduced to problem (4) in the case where the price functions are integrable, i.e.

$$
g_{i s}(w)=\frac{\partial \mu(w)}{\partial x_{i}}, i \in I_{s}, \text { and } h_{j s}(w)=-\frac{\partial \eta_{s}\left(w_{(s)}\right)}{\partial y_{j}}, j \in J_{s} ; s \in N .
$$

This is the case if these functions are separable, i.e. $g_{i s}(w)=g_{i s}\left(x_{i s}\right)$ for each $i \in I_{s}$ and $h_{j s}(w)=h_{j s}\left(y_{j s}\right)$ for each $j \in J_{s}$, for all $s \in N$. More precisely, VI (3) becomes the necessary optimality condition for (4). The reverse assertion is true if the functions $\mu$ and $\eta$ are convex.

We now describe an adaptive cyclic component-wise PL method for problem (6), which is some implementation of that from [15]. For each point $w \in W$ and each $s \in N$, we define by $V_{s}(w)$ the solution set of the optimization problem:

$$
\min _{v_{(s)} \in W_{s}} \rightarrow\left\{\left\langle v_{(s)}, \frac{\partial \mu(w)}{\partial w_{(s)}}\right\rangle+\eta_{s}\left(v_{(s)}\right)\right\} ;
$$

cf. (17). As above we suppose that the functions $\mu$ and $\eta$ are convex, $\mu$ is smooth, the set $W \subset \mathbb{R}^{m}$ is non-empty, convex, and compact. Under these assumptions $V_{s}(w)$ is also non-empty, convex, and compact. We define the gap function

$$
\varphi_{s}(w)=\max _{v_{(s)} \in W_{s}}\left\{\left\langle w_{(s)}-v_{(s)}, \frac{\partial \mu(w)}{\partial w_{(s)}}\right\rangle+\eta_{s}\left(w_{(s)}\right)-\eta_{s}\left(v_{(s)}\right)\right\}
$$


for each $s \in N$. For brevity, set $f(w)=\mu(w)+\eta(w)$ and denote by $\mathbb{Z}_{+}$the set of nonnegative integers. The optimal value of the function $f$ in (4) (or (6) ) will be denoted by $f^{*}$. The adaptive cyclic PL method is described as follows.

Method (CPL).

Initialization: Choose a point $z^{0} \in W$, numbers $\beta \in(0,1), \theta \in(0,1)$, and a sequence $\left\{\delta_{l}\right\} \searrow 0$. Set $l=1$.

Step 0: Set $k=0, d=0, s=1, w^{0}=z^{l-1}$.

Step 1: Solve problem (7), find $v_{(s)} \in V_{s}\left(w^{k}\right)$ and calculate $\varphi_{s}\left(w^{k}\right)$. If $\varphi_{s}\left(w^{k}\right) \geq \delta_{l}$, take

$$
p_{(i)}^{k}= \begin{cases}v_{(s)}-w_{(s)}^{k} & \text { if } i=s \\ \mathbf{0} & \text { if } i \neq s\end{cases}
$$

and go to Step 4 .

Step 2: Set $d=d+1$. If $d=n$, set $z^{l}=w^{k}, l=l+1$ and go to Step 0. (Restart)

Step 3: If $s=n$, set $s=1$, otherwise $s=s+1$. Afterwards go to Step 1 .

Step 4: Determine $j$ as the smallest number in $\mathbb{Z}_{+}$such that

$$
f\left(w^{k}+\theta^{j} p^{k}\right) \leq f\left(w^{k}\right)-\beta \theta^{j} \varphi_{s}\left(w^{k}\right),
$$

set $\lambda_{k}=\theta^{j}, w^{k+1}=w^{k}+\lambda_{k} p^{k}, k=k+1$. If $s=n$, set $s=1$, otherwise $s=s+1$. Afterwards go to Step 1.

Thus, the method has two levels. Each its outer iteration $l$ contains some number of inner iterations in $k$ with the sequential verification of descent value for each component with the fixed tolerance $\delta_{l}$. Completing each stage, which is marked as restart, leads to decreasing the tolerance value. The basic properties of CPL are deduced along the same lines as in [15].

Proposition 3.1 Suppose in addition that the gradient map the function $\mu$ is uniformly continuous on $W$. Then

(i) the number of inner iterations at each outer iteration $l$ is finite;

(ii) the sequence $\left\{z^{l}\right\}$ generated by Method (CPL) has limit points, all these limit points are solutions of problem ([6), besides,

$$
\lim _{l \rightarrow \infty} f\left(z^{l}\right)=f^{*} .
$$

The line-search procedure in the method admits various modifications. For instance, we can take the exact one-dimensional minimization rule instead of the current Armijo rule. If the gradient of the function $\mu$ is Lipschitz continuous, we can take fixed stepsize values and remove the line-search procedure at all; see [15] for more details.

Remark 3.1 Due to the presence of the control sequence $\left\{\delta_{l}\right\}, C P L$ differs essentially from the usual decomposition methods; see e.g. [14, 16]. At the same time, this technique is rather usual for for non-differentiable optimization methods; see e.g. [17]. It was also applied in iterative methods for linear inequalities [18] and for decomposable variational inequalities [19]. 


\section{A generalization of network equilibrium prob- lems with elastic demands}

We now consider network flow equilibrium problems with elastic (inverse) demands, which find various applications; see [20], [21, Chapter IV] and references therein.

Let us be given a graph with finite sets of nodes $\mathcal{M}$ and oriented $\operatorname{arcs} \mathcal{A}$ which join the nodes so that any arc $a=(i, j)$ has origin $i$ and destination $j$. Next, among all the pairs of nodes of the graph we extract a subset of origin-destination $(\mathrm{O} / \mathrm{D})$ pairs $\mathcal{N}$ of the form $s=(i \rightarrow j)$. Each pair $s \in \mathcal{N}$ is associated with the set of paths $\mathcal{P}_{s}$ which connect the origin and destination for this pair. Also, denote by $x_{p}$ the path flow for the path $p$. Given a flow vector $x=\left(x_{p}\right)_{p \in \mathcal{P}_{s}, s \in \mathcal{N}}$, one can determine the value of the arc flow

$$
f_{a}=\sum_{s \in \mathcal{N}} \sum_{p \in \mathcal{P}_{s}} \alpha_{p a} x_{p}
$$

for each arc $a \in \mathcal{A}$, where

$$
\alpha_{p a}= \begin{cases}1 & \text { if arc } a \text { belongs to path } p \\ 0 & \text { otherwise }\end{cases}
$$

If the vector $f=\left(f_{a}\right)_{a \in \mathcal{A}}$ of arc flows is known, one can determine the dis-utility value $c_{a}(f)$ for each arc. Then one can compute the dis-utility value for each path $p$ :

$$
g_{p}(x)=\sum_{a \in \mathcal{A}} \alpha_{p a} c_{a}(f)
$$

In the known elastic demand models, each $(\mathrm{O} / \mathrm{D})$ pair $s \in \mathcal{N}$ is associated with one variable value of flow demand and hence one inverse demand (dis-utility) function; see e.g. [21, Chapter IV] and references therein. However, many active agents (users) with different dis-utility functions may have the same physical location for many networks arising in applications. For this reason, we now consider the generalization, where each (O/D) pair $s \in \mathcal{N}$ may have several pairs of active users hence it is associated with the set of such pairs $\mathcal{B}_{s}$ so that each pair of users $j \in \mathcal{B}_{s}$ has its particular flow demand $y_{j}$ and dis-utility function $h_{j}$, which can be in principle dependent of the flow demand $y$, i.e. $y=\left(y_{j}\right)_{j \in \mathcal{B}_{s}, s \in \mathcal{N}}$. Then one can define the feasible set of flows:

$$
W=\left\{\begin{array}{l|l}
w=(x, y) \mid \begin{array}{c}
\sum_{p \in \mathcal{P}_{s}} x_{p}=\sum_{j \in \mathcal{B}_{s}} y_{j}, x_{p} \geq 0, p \in \mathcal{P}_{s} \\
0 \leq y_{j} \leq \gamma_{j}, j \in \mathcal{B}_{s} ; s \in \mathcal{N}
\end{array}
\end{array}\right\}
$$

We say that a feasible flow / demand pair $\left(x^{*}, y^{*}\right) \in W$ is an equilibrium point if it satisfies the following conditions:

$$
\forall s \in \mathcal{N}, \exists \lambda_{s} \text { such that } g_{p}\left(x^{*}\right)\left\{\begin{array}{ll}
\geq \lambda_{s} & \text { if } \quad x_{p}^{*}=0 \\
=\lambda_{s} & \text { if } \quad x_{p}^{*}>0
\end{array} \quad \forall p \in \mathcal{P}_{s}\right.
$$


and

$$
h_{j}\left(y^{*}\right) \begin{cases}\leq \lambda_{s} & \text { if } \quad y_{j}^{*}=0, \\ =\lambda_{s} & \text { if } \quad y_{j}^{*} \in\left(0, \gamma_{j}\right), \quad \forall j \in \mathcal{B}_{s} . \\ \geq \lambda_{s} & \text { if } \quad y_{j}^{*}=\gamma_{j},\end{cases}
$$

Clearly, the equilibrium conditions in (12)-(13) represent some implementation of the multi-commodity two-sided market equilibrium model (11)-(2), where each commodity is associated with an (O/D) pair $s \in \mathcal{N}$, its set of traders (carriers) with price functions $g_{p}(x)$ is represented by the paths $p \in \mathcal{P}_{s}$, whereas its set of buyers with price functions $h_{j}(y)$ is represented by the pairs of users $j \in \mathcal{B}_{s}$. We observe that the prices here are not fixed, the dependence of volumes for offer price functions $g_{p}$ is given in (8) -(10) and caused by the complexity of the system topology and by the fact that carriers of different $(\mathrm{O} / \mathrm{D})$ pairs can utilize the same links (arcs).

We now show that conditions (11) $-(13)$ can be equivalently rewritten in the form of a VI: Find a pair $\left(x^{*}, y^{*}\right) \in W$ such that

$$
\sum_{s \in \mathcal{N}} \sum_{p \in \mathcal{P}_{s}} g_{p}\left(x^{*}\right)\left(x_{p}-x_{p}^{*}\right)-\sum_{s \in \mathcal{N}} \sum_{j \in \mathcal{B}_{s}} h_{j}\left(y^{*}\right)\left(y_{j}-y_{j}^{*}\right) \geq 0 \quad \forall(x, y) \in W .
$$

Proposition 4.1 A pair $\left(x^{*}, y^{*}\right) \in W$ solves VI (14) if and only if it satisfies conditions (12)-(13).

Proof. Writing the usual necessary and sufficient optimality conditions (see [5, Proposition 11.7]) for problem (14), we obtain that there exist $x^{*} \geq \mathbf{0}, y^{*} \in[\mathbf{0}, \gamma]$, and $\lambda$ such that

$$
\begin{aligned}
& \sum_{p \in \mathcal{P}_{s}}\left(g_{p}\left(x^{*}\right)-\lambda_{s}\right)\left(x_{p}-x_{p}^{*}\right) \geq 0 \quad \forall x_{p} \geq 0, p \in \mathcal{P}_{s}, s \in \mathcal{N} ; \\
& \sum_{k \in \mathcal{B}_{s}}\left(\lambda_{s}-h_{j}\left(y^{*}\right)\right)\left(y_{j}-y_{j}^{*}\right) \geq 0 \quad \forall y_{j} \in\left(0, \gamma_{j}\right), s \in \mathcal{N} ; \\
& \sum_{p \in \mathcal{P}_{s}} x_{p}^{*}=\sum_{k \in \mathcal{B}_{s}} y_{j}^{*}, \quad j \in \mathcal{B}_{s}, s \in \mathcal{N} ;
\end{aligned}
$$

where $\lambda=\left(\lambda_{s}\right)_{s \in \mathcal{N}}$. However, the first and second relations are clearly equivalent to (12)-(13)

If each $(\mathrm{O} / \mathrm{D})$ pair is attributed to only one pair of users, we obtain the custom network equilibrium problems with elastic (inverse) demands; see e.g. [21, Chapter IV]. If all the $(\mathrm{O} / \mathrm{D})$ traffic demands in this model are not restricted with upper bounds, we obtain the model considered in [20]. Let us insert the same condition in our model:

$$
\gamma_{j}=+\infty \quad \forall j \in \mathcal{B}_{s}, s \in \mathcal{N} .
$$

Then (13) reduces to the following condition:

$$
h_{j}\left(y^{*}\right)\left\{\begin{array}{ll}
\leq \lambda_{s} & \text { if } \quad y_{j}^{*}=0, \\
=\lambda_{s} & \text { if } \quad y_{j}^{*}>0 ;
\end{array} \quad \forall j \in \mathcal{B}_{s} .\right.
$$


We can also write some other equivalent network equilibrium conditions, for instance,

$$
\begin{gathered}
g_{p}\left(x^{*}\right)-h_{j}\left(y^{*}\right)\left\{\begin{array}{lll}
=0 & \text { if } x_{p}^{*}>0 \quad \text { and } & y_{j}^{*}>0 \\
\geq 0 & \text { if } x_{p}^{*}=0 \quad \text { or } & y_{j}^{*}=0 ;
\end{array}\right. \\
\forall p \in \mathcal{P}_{s}, j \in \mathcal{B}_{s}, \quad s \in \mathcal{N} .
\end{gathered}
$$

Proposition 4.2 Let (15) hold. Then, for any pair $\left(x^{*}, y^{*}\right) \in W$, condition (17) is equivalent to (12) and (16).

Proof. Take an arbitrary pair $s \in \mathcal{N}$. Suppose a pair $\left(x^{*}, y^{*}\right) \in W$ satisfies conditions (12) and (16). Then, for any $p \in \mathcal{P}_{s}$ and $j \in \mathcal{B}_{s}$, the relations $x_{p}^{*}>0$ and $y_{j}^{*}>0$ imply $g_{p}\left(x^{*}\right)=\lambda_{s}=h_{j}\left(y^{*}\right)$. Next, each of the relations $x_{p}^{*}=0$ or $y_{j}^{*}=0$ implies $g_{p}\left(x^{*}\right) \geq \lambda_{s} \geq h_{j}\left(y^{*}\right)$, and (17) holds true.

Conversely, suppose a pair $\left(x^{*}, y^{*}\right) \in W$ satisfies conditions (17). Fix any $s \in \mathcal{N}$ and set

$$
\alpha^{\prime}=\min _{p \in \mathcal{P}_{s}} g_{p}\left(x^{*}\right), \alpha^{\prime \prime}=\max _{j \in \mathcal{B}_{s}} h_{j}\left(y^{*}\right),
$$

then $\alpha^{\prime} \geq \alpha^{\prime \prime}$. If $x_{p}^{*}=0$ for all $p \in \mathcal{P}_{s}$, then $y_{j}^{*}=0$ for all $j \in \mathcal{B}_{s}$ and conversely. Then taking any $\lambda_{s} \in\left[\alpha^{\prime \prime}, \alpha^{\prime}\right]$ yields (12) and (16). Otherwise, there exists at least one pair of indices $p \in \mathcal{P}_{s}, j \in \mathcal{B}_{s}$ such that $x_{p}^{*}>0$ and $y_{j}^{*}>0$. Then setting $\lambda_{s}=\alpha^{\prime}=\alpha^{\prime \prime}$ again yields (12) and (16).

It is easy to see that conditions (17) can be replaced with the following:

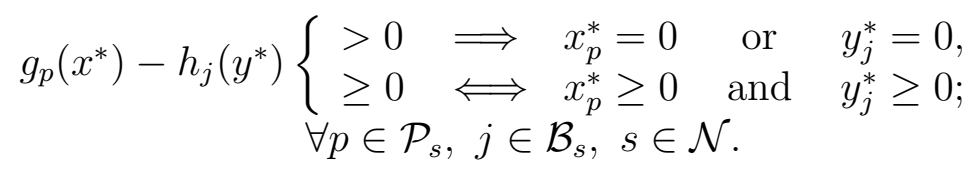

Proposition 4.3 Let (15) hold. Then, for any pair $\left(x^{*}, y^{*}\right) \in W$, condition (18) is equivalent to (12) and (16).

The equivalent VI formulation of network equilibrium problems enables us to obtain the existence of solutions rather easily. The feasible set $W$ of the network equilibrium problem defined in (11) is bounded if $\gamma_{j}<+\infty$ for all $j \in \mathcal{B}_{s}, s \in \mathcal{N}$. Then VI (14) and hence the equivalent network equilibrium problem are solvable if all the mappings $c_{a}$, $a \in \mathcal{A}$ and $h_{j}, j \in \mathcal{B}_{s}, s \in \mathcal{N}$ are continuous. Let us turn to the above pure unbounded case (15). Then the feasible set $W$ in (11) is unbounded. We now deduce a new existence result for VI (14) and hence for the equivalent network equilibrium problem by a direct application of Proposition 2.2. We need the proper following coercivity condition; cf. (C).

(C1) There exists a number $r>0$ such that for any point $w=(x, y) \in W$ and for each $s \in \mathcal{N}$ it holds that

$$
\exists j \in \mathcal{B}_{s}, y_{j}>r \Longrightarrow \exists p \in \mathcal{P}_{s} \text { such that } x_{p}>0 \text { and } g_{p}(x) \geq h_{j}(y) .
$$

We observe that condition (C1) implies condition (C) for VI (14) and we obtain the desired existence result. 
Theorem 4.1 Suppose that (15) holds, the set $W$ defined in (11) is nonempty, all the functions $c_{a}$ and $h_{j}$ are continuous for all $a \in \mathcal{A}, j \in \mathcal{B}_{s}$, and $s \in \mathcal{N}$. If condition (C1) is fulfilled, then VI (14) has a solution.

\section{Implementation of partial linearization methods for integrable network equilibrium problems}

In Section 3, several versions of partial linearization (PL) methods for special decomposable optimization problems over Cartesian product sets were described for the general multi-commodity market equilibrium model of Section 2 in the integrable case. Hence, PL methods can be also applied to integrable network equilibrium problems with elastic demands of Section 4.

Therefore, we now will suppose that all the functions $c_{a}$ and $h_{j}$ are continuous and separable, i.e., $c_{a}(f)=c_{a}\left(f_{a}\right)$ and $h_{j}(y)=h_{j}\left(y_{j}\right)$. Besides, we assume that $c_{a}\left(f_{a}\right)$ and $-h_{j}\left(y_{j}\right)$ are monotone increasing functions. Next, we assume that

$$
\gamma_{j}<+\infty \quad \forall j \in \mathcal{B}_{s}, s \in \mathcal{N} ;
$$

then the feasible set $W$ is non-empty, convex, and compact and

$$
\begin{aligned}
W= & \prod_{s \in \mathcal{N}} W_{s}, \text { where } \\
W_{s}= & \begin{cases}\left.w_{(s)}=\left(x_{(s)}, y_{(s)}\right) \mid \begin{array}{l}
\sum_{p \in \mathcal{P}_{s}} x_{p}=\sum_{j \in \mathcal{B}_{s}} y_{j}, \\
x_{p} \geq 0, p \in \mathcal{P}_{s}, 0 \leq y_{j} \leq \gamma_{j}, j \in \mathcal{B}_{s}
\end{array}\right\} ; \\
& \text { for } s \in \mathcal{N} .\end{cases}
\end{aligned}
$$

Here $x_{(s)}=\left(x_{p}\right)_{p \in \mathcal{P}_{s}}, y_{(s)}=\left(y_{j}\right)_{j \in \mathcal{B}_{s}}$.

Due to the separability of the functions $c_{a}$ and $h_{j}$, their continuity implies integrability, i.e., then there exist functions

$$
\mu_{a}\left(f_{a}\right)=\int_{0}^{f_{a}} c_{a}(t) d t \forall a \in \mathcal{A}, \eta_{j}\left(y_{j}\right)=\int_{0}^{v_{j}} h_{j}(t) d t \forall j \in \mathcal{B}_{s}, s \in \mathcal{N} .
$$

Taking into account (8), we see that VI (14) gives a necessary and sufficient optimality condition for the following optimization problem:

$$
\min _{(x, y) \in W} \rightarrow\left\{\sum_{a \in \mathcal{A}} \mu_{a}\left(f_{a}\right)-\sum_{s \in \mathcal{N}} \sum_{j \in \mathcal{B}_{s}} \eta_{j}\left(y_{j}\right)\right\} .
$$

However, this problem falls into the basic format (6) and the suggested PL methods can be applied to (19). 
We describe the solution of the basic direction finding problem (7). It now consists in finding an element $\bar{w}_{(s)}=\left(\bar{x}_{(s)}, \bar{y}_{(s)}\right) \in W_{s}$, which solves the optimization problem

$$
\min _{\left(x_{(s)}, y_{(s)}\right) \in W_{s}} \rightarrow\left\{\sum_{p \in \mathcal{P}_{s}} g_{p}\left(x^{k}\right) x_{p}-\sum_{j \in \mathcal{B}_{s}} \eta_{j}\left(y_{j}\right)\right\}
$$

for some selected pair $s \in \mathcal{N}$. The solution of (20) can be found with the simple procedure below, which is based on optimality conditions (12)-(13).

First we calculate the shortest path $q \in \mathcal{P}_{s}$ with the minimal cost. Set $\tilde{\lambda}_{s}=g_{q}\left(x^{k}\right)$, $\bar{x}_{p}=0$ for all $p \in \mathcal{P}_{s}$.

For each $j \in \mathcal{B}_{s}$ we verify three possible cases.

Case 1. If $h_{j}(0) \leq \tilde{\lambda}$, then set $\bar{y}_{j}=0$. Otherwise go to Case 2 .

Case 2. If $h_{j}\left(\gamma_{j}\right) \geq \tilde{\lambda}$, set $\bar{y}_{j}=\gamma_{j}, \bar{x}_{q}=\bar{x}_{q}+\gamma_{j}$. Otherwise go to Case 3 .

Case 3. We have $h_{j}\left(\gamma_{j}\right)<\tilde{\lambda}<h_{j}(0)$. By continuity of $h_{j}$, we find the value $\bar{y}_{j} \in\left[0, \gamma_{j}\right]$ such that $h_{j}\left(\bar{y}_{j}\right)=\tilde{\lambda}$, set $\bar{x}_{q}=\bar{x}_{q}+\bar{y}_{j}$.

Therefore, the suggested PL methods can be implemented rather easily.

\section{Application of market models to resource alloca- tion in wireless networks}

In contemporary wireless networks, increasing demand of services leads to serious congestion effects, whereas significant network resources (say, bandwidth and batteries capacity) are utilized inefficiently for systems with fixed allocation rules. This situation forces one to apply more flexible market type allocation mechanisms. Due to the presence of conflict of interests, most papers on allocation mechanisms are devoted to pure game-theoretic models reflecting imperfect competition; see, e.g., [22, 23]. However, certain lack of information about the participants is typical for wireless telecommunication networks (see, e.g., [24, 23]), and some other market models may be suitable here because they can be utilized under minimal information requirements on involved users.

We now consider the problem of allocation of services of several competitive wireless network providers for a large number of users, which is very essential for contemporary communication systems. This problem was investigated in [25, 26, 27, 28] for wired and wireless network settings, where game-theoretic models for competitive providers were presented. An alternative model, which is based on some VI formulation and uses proper equilibrium conditions, was suggested for this problem in [29, Section 6]. We now propose its extension that admits different kinds of users' behavior.

Namely, we suppose that there are $m$ wireless network providers and that all the users are divided into $n$ classes, that is, the users belonging to the same class $j$ are considered as one service consumer with a price function $h_{j}\left(y_{j}\right)$ and a scalar bid volume $y_{j} \in\left[0, \beta_{j}\right]$ for $j \in N=\{1, \ldots, n\}$. Next, each provider $i$ announces his/her price 
function $b_{i}\left(x_{i}\right)$ depending on the offer volume $x_{i} \in\left[0, \alpha_{i}\right]$ for $i \in M=\{1, \ldots, m\}$. However, such joint consumption of wireless network resources yields the additional dis-utility $l_{i}(x)$ for users consuming resources of provider $i$, where $x=\left(x_{1}, \ldots, x_{s}\right)^{\top}$; see [26, 27, 28] for more detail. Hence, the actual price function of provider $i$ for users becomes $g_{i}(x)=b_{i}\left(x_{i}\right)+l_{i}(x)$. We can thus define the feasible set of offer/bid values

$$
D=\left\{\begin{array}{l|l}
(x, y) \mid \begin{array}{l}
x_{i} \in\left[0, \alpha_{i}\right], i \in M, \\
y_{j} \in\left[0, \beta_{j}\right], j \in N ;
\end{array} \sum_{i \in M} x_{i}=\sum_{j \in N} y_{j} ;
\end{array}\right\}
$$

where $y=\left(y_{1}, \ldots, y_{n}\right)^{\top}$. Then we can write the two-sided equilibrium problem that consists in finding a feasible pair $(\bar{x}, \bar{y}) \in D$ and a price $\lambda$ such that

$$
g_{i}(\bar{x})\left\{\begin{array}{l}
\geq \lambda, \text { if } \bar{x}_{i}=0, \\
=\lambda, \text { if } \bar{x}_{i} \in\left(0, \alpha_{i}\right), \quad h_{j}\left(\bar{y}_{j}\right)\left\{\begin{array}{l}
\leq \lambda, \text { if } \bar{y}_{j}=0, \\
\leq \lambda, \text { if } \bar{x}_{i}=\alpha_{i},
\end{array} \quad \text { if } \bar{y}_{j} \in\left(0, \beta_{j}\right),\right. \\
\geq \lambda, \text { if } \bar{y}_{j}=\beta_{j},
\end{array}\right.
$$

Clearly, it is a particular case of those in (11)-(2). Due to Proposition 2.1, (21) can be replaced with the equivalent VI: Find $(\bar{x}, \bar{y}) \in D$ such that

$$
\sum_{i \in M} g_{i}(\bar{x})\left(x_{i}-\bar{x}_{i}\right)-\sum_{j \in N} h_{j}\left(\bar{y}_{j}\right)\left(y_{j}-\bar{y}_{j}\right) \geq 0 \quad \forall(x, y) \in D .
$$

This property enables us to establish existence of solutions for the above problem and develop efficient iterative solution methods. In fact, if all the price functions are continuous and the set $D$ is nonempty and bounded, then VI (22) has a solution. In the unbounded case, some coercivity condition is necessary. For instance, let us consider the case where $\alpha_{i}=+\infty$ for $i \in M$ and $\beta_{j}=+\infty$ for $j \in N$ and take the following condition; cf. (C).

(C2) There exists a number $r>0$ such that for any pair $(x, y) \in D$ it holds that

$$
y_{l}>r \Longrightarrow \exists k \in M \text { such that } x_{k}>0 \text { and } g_{k}(x) \geq h_{l}\left(y_{l}\right) .
$$

Clearly, (C2) implies (C) for VI (22) and Proposition 2.2 provides the existence result.

Theorem 6.1 Suppose that the set D is nonempty, the functions $g_{i}$ and $h_{j}$ are continuous for all $i \in M, j \in N$. If condition (C2) is fulfilled, then VI (22) has a solution.

\section{The partial linearization method for resource al- location problems in wireless networks}

Iterative solution methods for solving VI of form (22) in general require additional monotonicity assumptions for convergence; see e.g. [14, 5, 10]. Additional solution 
methods appear in the integrable case where

$$
g_{i}(x)=\frac{\partial \mu(x)}{\partial x_{i}}, i \in M ; h_{j}\left(y_{j}\right)=-\eta_{j}^{\prime}\left(y_{j}\right), j \in N
$$

Then, VI (22) gives the optimality condition for the optimization problem:

$$
\begin{gathered}
\min _{w \in D} \rightarrow f(w), \\
f(w)=f(x, y)=\{\mu(x)+\eta(y)\}, \eta(y)=\sum_{j \in N} \eta_{j}\left(y_{j}\right)
\end{gathered}
$$

cf. (44) and (6). In particular, conditional gradient, gradient projection, and Uzawa type methods then can be utilized; see e.g. [7, 8]. We now only describe a way to implement the custom PL method since the problem is not separable. We suppose in addition that the function $\mu$ is smooth and convex, $\alpha_{i}=+\infty$ for all $i \in M$, and $0 \leq \beta_{j}<+\infty$ for all $j \in N$. Then the feasible set $D$ is non-empty, convex, and compact.

For more clarity, we rewrite the PL method for problem (23). We define the gap function

$$
\varphi(w)=\varphi(x, y)=\max _{\left(x^{\prime}, y^{\prime}\right) \in D}\left\{\left\langle x-x^{\prime}, \mu^{\prime}(x)\right\rangle+\eta(y)-\eta\left(y^{\prime}\right)\right\}
$$

\section{Method (PL).}

Choose a point $w^{0} \in D$, numbers $\beta \in(0,1)$ and $\theta \in(0,1)$, set $k=0$. At the $k$-th iteration, $k=0,1, \ldots$, we have a point $w^{k} \in D$. Find a solution $v^{k}=\left(\bar{x}^{k}, \bar{y}^{k}\right)$ of the problem

$$
\min _{v \in D} \rightarrow\left\{\left\langle\mu^{\prime}\left(x^{k}\right), v\right\rangle+\eta(v)\right\}
$$

If $v^{k}=w^{k}$, stop. Otherwise set $d^{k}=v^{k}-w^{k}$, find $p$ as the smallest number in $\mathbb{Z}_{+}$such that

$$
f\left(w^{k}+\theta^{p} d^{k}\right) \leq f\left(w^{k}\right)-\beta \theta^{p} \varphi\left(w^{k}\right),
$$

set $\sigma_{k}=\theta^{p}, w^{k+1}=w^{k}+\sigma_{k} d^{k}$, and $k=k+1$.

The solution of the basic direction finding problem (24) can also be found with the simple procedure, which is similar to that from Section 5 and based on the optimality conditions.

First we calculate an index $q \in M$ that corresponds to the minimal value

$$
g_{q}\left(x^{k}\right)=\min _{i \in M} g_{i}\left(x^{k}\right)
$$

and set $\tilde{\lambda}=g_{q}\left(x^{k}\right), \bar{x}_{i}^{k}=0$ for all $i \in M$.

For each $j \in N$ we verify three possible cases.

Case 1. If $h_{j}(0) \leq \tilde{\lambda}$, then set $\bar{y}_{j}^{k}=0$. Otherwise go to Case 2 . 
Case 2. If $h_{j}\left(\beta_{j}\right) \geq \tilde{\lambda}$, set $\bar{y}_{j}^{k}=\beta_{j}, \bar{x}_{q}^{k}=\bar{x}_{q}^{k}+\beta_{j}$. Otherwise go to Case 3 .

Case 3. We have $h_{j}\left(\beta_{j}\right)<\tilde{\lambda}<h_{j}(0)$. By continuity of $h_{j}$, we find the value $\bar{y}_{j}^{k} \in\left[0, \beta_{j}\right]$ such that $h_{j}\left(\bar{y}_{j}^{k}\right)=\tilde{\lambda}$, set $\bar{x}_{q}^{k}=\bar{x}_{q}^{k}+\bar{y}_{j}^{k}$.

Let us now consider the case where $0 \leq \alpha_{i}<+\infty$ for all $i \in M$ and $0 \leq \beta_{j}<+\infty$ for all $j \in N$. Then the feasible set $D$ is also non-empty, convex, and compact. Hence, the above PL method can be applied to (23), however, we should then take more complex procedures for solution of problem (24). However, we can eliminate the upper bounds for the variables $x_{i}$ via a suitable penalty approach.

For instance, replace problem (23) with the sequence of auxiliary problems of the form

$$
\begin{gathered}
\min _{w \in D} \rightarrow \Phi(w, \tau), \\
\Phi(w, \tau)=\mu(x)+\tau \varphi(x)+\eta(y), \varphi(x)=0.5 \sum_{i \in M} \max \left\{x_{i}-\alpha_{i}, 0\right\}^{2}
\end{gathered}
$$

where $\tau>0$ is a penalty parameter, the functions $\mu$ and $\eta$ are defined as above. Under the standard assumptions the sequence of solutions of (25) will approximate a solution of (23) if $\tau \rightarrow+\infty$; see e.g. [10]. Next, each problem (25) has the previous format without the upper bounds for the variables $x_{i}$. Hence, we can apply directly the above version of the PL method to (25) with replacing $f(w)$ by $\Phi(w, \tau)$. Clearly, (24) is replaced by

$$
\min _{v \in D} \rightarrow\left\{\left\langle\mu^{\prime}\left(x^{k}\right)+\tau \varphi^{\prime}\left(x^{k}\right), v\right\rangle+\eta(v)\right\} .
$$

We also have to substitute each function $g_{i}(x)$ with $\tilde{g}_{i}(x)=g_{i}(x)+\tau \max \left\{x_{i}-\alpha_{i}, 0\right\}$ in the procedure of finding its solution. This gives us an alternative way to solve such resource allocation problems in wireless networks.

\section{Computational experiments with network equi- librium test problems}

In order to compare the performance of the PL methods we carried out preliminary series of computational experiments on network equilibrium test problems of form (11) -(13) or (14). We took their adjustment described in Section 5.

For comparison we took proper extensions of the known test examples of network equilibrium problems with elastic demands, namely, each $(\mathrm{O} / \mathrm{D})$ pair was associated with two pairs of active users. We used the arc cost functions $c_{a}\left(f_{a}\right)=1+f_{a}$ for all $a \in \mathcal{A}$ and the minimal path cost (dis-utility) functions $h_{j 1(s)}\left(y_{j 1}\right)=30-0.5 y_{j 1(s)}$ and $h_{j 2(s)}\left(y_{j 2(s)}\right)=28-0.3 y_{j 2(s)}$, where $\mathcal{B}_{s}=\{j 1(s), j 2(s)\}$ for all $s \in \mathcal{N}$. We took

$$
\Delta_{k}=\varphi\left(w^{k}\right)=\sum_{s \in \mathcal{N}} \varphi_{s}\left(w^{k}\right)
$$


as accuracy measure for the methods. Both the PL and CPL methods were implemented with the Armijo line-search rule where $\beta=\theta=0.5$. Due to the above description we see that we can take the total number of blocks where the linesearch procedure was utilized as unified complexity measure for both the methods, which will be called block iterations. Hence we reported this value in the tables for attaining different accuracies. The methods were implemented in $\mathrm{C}++$ with double precision arithmetic.

The topology of Example 1 was taken from [30]. The graph contains 25 nodes, 40 arcs, and $5 \mathrm{O} / \mathrm{D}$ pairs. We used two rules for changing the parameter $\delta_{l}$ with $\delta_{0}=10$ in CPL. The performance results are given in Table 1.

Table 1: Example 1. The numbers of block iterations

\begin{tabular}{|r|c|c|c|}
\hline accuracy & PL & CPL & CPL \\
\hline & & $\delta_{l+1}=\delta_{l} / 2$ & $\delta_{l}=\delta_{0} / l$ \\
\hline 0.2 & 4970 & 4427 & 3519 \\
\hline 0.1 & 10785 & 8747 & 6411 \\
\hline 0.05 & 21260 & 17284 & 13425 \\
\hline
\end{tabular}

The topology of Example 2 was taken from [31, Network 26]. The graph contains 22 nodes, 36 arcs, and $12 \mathrm{O} / \mathrm{D}$ pairs. We used the rule $\delta_{l}=\delta_{0} / l$ with $\delta_{0}=10$ in CPL. The performance results are given in Table 2 .

Table 2: Example 2. The numbers of block iterations

\begin{tabular}{|r|c|c|}
\hline accuracy & PL & CPL \\
\hline 0.2 & 420 & 233 \\
\hline 0.1 & 468 & 246 \\
\hline 0.05 & 504 & 256 \\
\hline
\end{tabular}

In Example 3, the data were generated randomly. The graph contained 20 nodes, 114 arcs, and $10 \mathrm{O} / \mathrm{D}$ pairs. We used the rule $\delta_{l}=\delta_{0} / l$ with $\delta_{0}=10$ in $\mathrm{CPL}$. The results are given in Table 3. In all the cases, CPL showed certain preference over PL in the number of block iterations.

\section{Conclusions}

We considered the general market model with many divisible commodities and price functions of participants and established existence results for this problem under natural coercivity conditions in the case of an unbounded feasible set. We described 
Table 3: Example 3. The numbers of block iterations

\begin{tabular}{|r|r|r|}
\hline accuracy & PL & CPL \\
\hline 1 & 135730 & 106308 \\
\hline 0.5 & 271830 & 217932 \\
\hline 0.2 & 662220 & 531032 \\
\hline 0.1 & 1329910 & 1082449 \\
\hline
\end{tabular}

extensions of the known network flow equilibrium problems with elastic demands and a resource allocation problem in wireless communication networks and showed they are particular cases of the presented market model. This property enabled us to obtain new existence results for all these models as some adjustments of that for the general market model. Besides, under certain integrability conditions the market model can be reduced to an optimization problem. We suggested a new cyclic version of the partial linearization (PL) method for its decomposable case. We suggested ways for implementation of the PL method to solve the network equilibrium problems and resource allocation problems in wireless communication networks.

\section{Acknowledgements}

This work was supported by the RFBR grant, project No. 16-01-00109a and by grant No. 297689 from Academy of Finland. The author is grateful to Olga Pinyagina for her assistance in carrying out computational experiments.

\section{References}

[1] Nikaido, H.: Convex Structures and Economic Theory. Academic Press, New York (1968)

[2] Okuguchi, K., Szidarovszky, F.: The Theory of Oligopoly with Multi-product Firms. Springer-Verlag, Berlin (1990)

[3] Nash, J.: Non-cooperative games. Ann. Math., vol.54, 286-295 (1951)

[4] Konnov, I.V.: On modeling of auction type markets. Issled. Inform., vol.10, 73-76 (2006) [in Russian]

[5] Konnov, I.V.: Equilibrium Models and Variational Inequalities. Elsevier, Amster$\operatorname{dam}(2007)$ 
[6] Konnov, I.V.: On variational inequalities for auction market problems. Optim. Lett., vol.1, 155-162 (2007)

[7] Konnov, I.V.: Equilibrium models for multi-commodity auction market problems. Adv. Model. and Optim., vol.15, 511-524 (2013)

[8] Konnov, I.V.: An alternative economic equilibrium model with different implementation mechanisms. Adv. Model. Optim. vol.17, 245-265 (2015)

[9] Konnov, I.V.: On vector formulations of auction-type problems with applications. Optimization, vol.65, 233-251 (2016)

[10] Konnov, I.V.: Nonlinear Optimization and Variational Inequalities. Kazan Univ. Press, Kazan (2013) [In Russian]

[11] Mine, H., Fukushima, M.: A minimization method for the sum of a convex function and a continuously differentiable function. J. Optim. Theory Appl., vol.33, 9-23 (1981)

[12] Patriksson, M.: Cost approximation: a unified framework of descent algorithms for nonlinear programs. SIAM J. Optim., vol.8, 561-582 (1998)

[13] Bredies, K., Lorenz, D.A., Maass, P.: A generalized conditional gradient method and its connection to an iterative shrinkage method. Comput. Optim. Appl., vol. 42, 173-193 (2009)

[14] Patriksson, M.: Nonlinear Programming and Variational Inequality Problems: A Unified Approach. Kluwer Academic Publishers, Dordrecht (1999)

[15] Konnov, I.V.: An adaptive partial linearization method for optimization problems on product sets. arXiv:1605.01971v2. http://arxiv.org/abs/1605.01971 (Accessed 25 May 2016)

[16] Migdalas, A.: Cyclic linearization and decomposition of team game models. In: Butenko, S., Murphey, R., Pardalos, P. (eds.) Recent Developments in Cooperative Control and Optimization. Kluwer Academic Publishers, Dordrecht, 332-348 (2004)

[17] Balinski, M.L., Wolfe, P.: (ed.) Nondifferentiable Optimization. Math. Progr. Study 3. North - Holland, Amsterdam, (1975)

[18] McCormick, S.F.: The methods of Kaczmarz and row orthogonalization for solving linear equations and least squares problems in Hilbert space. Indiana Univ. Math. J., vol. 26, 1137-1150 (1977)

[19] Konnov, I.V.: A class of combined relaxation methods for decomposable variational inequalities. Optimization, vol. 51, 109-125 (2002) 
[20] Dafermos, S.: The general multimodal network equilibrium problem with elastic demand. Networks, vol.12, 57-72 (1982)

[21] Nagurney, A.: Network Economics: A Variational Inequality Approach. Kluwer, Dordrecht (1999)

[22] Leshem, A., Zehavi, E.: Game theory and the frequency selective interference channel: A practical and theoretic point of view. IEEE Signal Process., vol.26, 28-40 (2009)

[23] Raoof, O., Al-Raweshidy, H.: Auction and game-based spectrum sharing in cognitive radio networks. In: Huang, Q., (ed.), Game Theory, Sciyo, Rijeka, Ch. 2, $13-40(2010)$

[24] Iosifidis, G., Koutsopoulos, I.: Double auction mechanisms for resource allocation in autonomous networks. IEEE J. Sel. Areas Commun., vol.28, 95-102 (2010)

[25] Hayrapetyan, A., Tardos, É., Wexler, T., A network pricing game for selfish traffic. Distrib. Comput., vol.19, 255-266 (2007)

[26] Korcak, O., Iosifidis, G., Alpcan, T., Koutsopoulos, I.: Competition and regulation in a wireless operators market: an evolutionary game perspective. In: Proc. 6th Int. Conf. on Network Games, Control and Optim. (NETGCOOP), Avignon, IEEE $17-24(2012)$

[27] Maillé, P., Tuffin, B., Vigne, J.M.: Competition between wireless service providers sharing a radio resource. In: Proc. 11th IFIP Networking Conference. Part II. Springer, Berlin, 355-365 (2012)

[28] Zhang, F., Zhang, W.: Competition between wireless service providers: Pricing, equilibrium and efficiency. In: 11th International Symposium and Workshops on Modeling and Optimization in Mobile, Ad Hoc and Wireless Networks (WiOpt 2013), IEEE, 208-215 (2013)

[29] Konnov I.V.: On auction equilibrium models with network applications. Netnomics, vol. 16, 107-125 (2015)

[30] Bertsekas, D., Gafni, E.: Projection methods for variational inequalities with application to the traffic assignment problem. Math. Progr. Study, vol. 17, 139159 (1982)

[31] Nagurney, A.: Comparative tests of multi-modal traffic equilibrium problems. Transp. Sci. B, vol. 18, 469-485 (1984) 